\title{
Model of high-temperature plastic deformation of nanocrystalline materials: Application to yttria tetragonal zirconia
}

\begin{abstract}
D. Gómez-García, C. Lorenzo-Martín, A. Muñoz-Bernabé, and A. Domínguez-Rodríguez*
Departamento de Física de la Materia Condensada, Universidad de Sevilla, Apto. 1065, 41080 Sevilla, Spain

(Received 23 October 2002; revised manuscript received 21 January 2003; published 1 April 2003)

The possibility of the influence of segregation-induced local electric fields in the bulk diffusion of the species controlling the plastic deformation of nanocrystalline materials has been pointed out. Until now, there is only a model applicable to the case of a monodimensional system. In spite of its simplicity, it predicts a significative influence of a local electric field in creep. Our work develops a different model applicable to three-dimensional systems. It takes as a starting point the diffusional model, and it can be generalized to those systems in which the grain-boundary sliding model accommodated by diffusional processes accurately describes plasticity in the submicron range of grain size. The range of validity, as well as the different behavior of nanocrystalline materials from the submicron ones is discussed. Preliminary results are in good agreement with the published data for yttria tetragonal zirconia (YTZP) nanocrystalline ceramics.
\end{abstract}

DOI: 10.1103/PhysRevB.67.144101

PACS number(s): 62.25.+g, 62.20.Hg, 62.20.Fe

\section{INTRODUCTION}

Nanocrystalline materials are a field of very active research activity in materials science. From a practical point of view, miniaturization is a basic requirement to continue the rapid improvement of electronic devices in the 21st Century. ${ }^{1}$ This effort is concentrated not only on metallic nanocrystalline systems, but also on nonmetallic ones, particularly ceramics to be used in industry under extreme conditions. Regarding ceramics, another main goal is the search for the optimum conditions for processing of complex pieces, i.e., superplastic ceramic materials capable of being deformed at high strain rates and at moderate temperatures. Nanoceramics can play a crucial role, as it is supposed that their small grain sizes can induce high strain-rate plasticity, without loss of the favorable properties of ceramics. ${ }^{2}$

In the case of nanocrystalline ceramics the modeling of the deformation mechanisms controlling their plasticity is critical to understanding and tailoring microstructures to meet mattering requirements. This is a very difficult and challenging task, because the leading influence of grain boundaries is difficult to model. In particular, in many ceramic materials, grain boundaries are sinks and sources of some chemical species, whose influence in creep and diffusion can be crucial. This work develops a model to explain the high-temperature creep behavior of nanoceramics in which grain-boundary segregation of some chemical species is important.

The existence of segregation of charged chemical ions produces a local electric field; as a consequence, ion diffusion should be significatively affected. As diffusion is a basic ingredient of the principal high-temperature deformation mechanisms, changes in the effective diffusion coefficient of the different diffusing species can be detected by their effects on plasticity. The influence of those local electric fields should become more significant for smaller grain sizes.

$\mathrm{A} \mathrm{model}^{3}$ exists to relate diffusion and deformation, but it uses as a starting point a monodimensional system in which grain boundaries are treated as infinite thin films. Diffusion takes place along a direction normal to the interface. This simplification permits the exact resolution of both diffusion and electric-field equations. Some of the hypotheses considered in that model have been saved in ours, although we have tried to give a step forward to a realistic threedimensional one, and consequently, some other improvements have been made.

No model is really useful unless it can account for the properties of real materials. In this paper we have focused on the explanation of the high-temperature creep behavior of yttria tetragonal zirconia polycrystals (YTZP's) whose grain sizes range from submicron to the nanometer scale. There are ample publications on the high-temperature plasticity features of submicron-sized YTZP ceramics (for a review, see Refs. 4 and 5). However, when going down to nanometer size, the number of publications decreases. This is mainly due to the fact that well densified polycrystals have been available only very recently. Domínguez-Rodríguez et al. ${ }^{6}$ and Gutiérrez-Mora, Jiménez-Melendo, DomínguezRodríguez, and Chaim ${ }^{7}$ have recently reported creep data on fully dense nano-YTZP. Quiet astonishingly, YTZP nanocrystals seem to exhibit an improved creep resistance when compared with that of the submicron-sized ones; (i.e., the strain rate is lower than expected by extrapolation to the nanometric scale of the results measured in submicrometric specimens). In addition to that, the strain rate has a powerlaw dependence with the mean grain size, with an exponent close to -1 instead of -2 , as would be expected from a volume diffusion controlled creep mechanism. A strong temperature dependence is found, with an activation energy for diffusion much higher compared with the activation energy for bulk cationic diffusion. These properties are in disagreement with the values reported for plastic deformation in the submicron-ranged YTZP ceramics (the power-law dependence of the strain rate with the mean grain size fits to an exponent -2 and the activation energy is that of bulk cationic diffusion).

On the other hand, the existence of yttrium segregation at 
the grain boundaries in YTZP polycrystals is well documented. $^{8-11}$ Conrad $^{12}$ and Conrad and Yang ${ }^{13}$ for the model-case of $\mathrm{MgO}$ have pointed out the possible role of segregation in the mechanical properties of oxides, particularly when they are nanostructured. As a consequence of all of this, YTZP is revealed as an excellent system in which the main predictions of our model can be developed and compared with experiment.

The main features of our model will be presented in the following section. As the applicability of the model will be tested in YTZP nanoscaled ceramics, our main assumptions will be always referred to the case of this particular ceramic system.

\section{MODELING}

\section{A. Grain-boundary segregation and local electric field}

The most important driving force for dopant segregation to interfaces is the strain energy relaxation. The existence of segregation of dopants whose electric charge is different from that of the parent ions accounts for the existence of local electric fields. In the case of YTZP the accumulation of yttrium ions at the interface ${ }^{14,15}$ induces a local density of negative charge produced by the $Y_{\mathrm{Zr}}^{\prime}$ defects. This local electric field produces a gradient of the oxygen vacancies between the grain bulk and the boundaries. These oxygen vacancies have a screening effect on the electric field. In consequence, once a critical depth, close to the boundaries, is reached inside the grain (in which the oxygen vacancy concentration is higher than that in the bulk) the electric field becomes negligible. According to electrodynamics, the thickness of the layer where the electric field is not zero is known as the Debye attenuation length. From now on, we will label that distance as $\lambda$. For most materials $\lambda$ at room temperature it is in between 1 and $10 \mathrm{~nm}$ (see Ref. 3).

The picture described above may be significantly altered when amorphous integranular phases are present. This is due to the possible additional screening effect of those phases. In what follows, no glassy phases will be supposed to exist.

\section{B. Electric potential inside the grains}

The electric potential inside the grain is a complicated function depending on the boundary conditions, i.e., the grain shape, and an analytical expression is not possible in the general case. Therefore our model will consist of a polycrystal of perfectly spherical grains whose radius is $R$. In a real crystal, grains cannot be perfectly spherical, because such a microstructure is not a fully dense spatial configuration. Real grains are polyhedral. However, a spherical grain will be an "average" approximation to a polyhedral one with many faces. In real grains, the local electric field is a function of the three spherical coordinates: $r, \theta$, and $\phi$. The spherical grain is the limit case in which the local electric field $\mathbf{E}(r, \theta, \phi)$ is given by

$$
\langle\mathbf{E}(r)\rangle=\frac{1}{4 \pi} \int_{0}^{2 \pi} d \phi \int_{0}^{\pi} d \theta \sin \theta \mathbf{E}(r, \theta, \phi),
$$

i.e., the electric field in a spherical grain is the resultant field when all the orientational dependencies have been averaged: $\langle\mathbf{E}(r)\rangle$. The polar axis is chosen as the loading direction. Consequently, this field is spherically symmetric.

In accordance with this symmetry, several implications can be made: (i) The charge density $\rho(r)$ at the grain boundaries will be spherically symmetric. Assuming that the segregation layer is thin enough the charge density can be modeled by a step function:

$$
\rho(r)= \begin{cases}0 & \text { if } 0 \leqslant r<R-\lambda, \\ \rho & \text { if } R-\lambda \leqslant r \leqslant R .\end{cases}
$$

(ii) Grains can be treated as isolated when calculating the electric field inside them. This is due to the fact that intergranular space is charged with an opposite charge in order to guarantee the neutrality of the system. Our system can be pictured as a set of spherical grains with a segregation layer at their boundaries embedded in a matrix with opposite charge.

According to classical electrodynamics, ${ }^{16}$ the electric potential $V(r)$ is related to the charge density through the Poisson equation for spherically symmetric systems:

$$
\frac{1}{r^{2}} \frac{\partial}{\partial r}\left(r^{2} \frac{\partial V}{\partial r}\right)=-\frac{\rho(r)}{\varepsilon},
$$

where $\varepsilon$ is the dielectric constant of the material under study. The Poisson equation can be solved according to standard procedures of electrodynamics: both the electric field and the electric potential must be continuous functions and the electric potential vanishes very far from the grain boundary. Once those boundary conditions are taken into account, the following mathematical solutions are achieved:

$$
V(r)= \begin{cases}\frac{\rho R^{2}}{6 \varepsilon}-\frac{\rho(R-\lambda)^{2}}{2 \varepsilon}+\frac{\rho(R-\lambda)^{3}}{3 \varepsilon R}+\frac{\rho}{3 \varepsilon_{0} R}\left[R^{3}-(R-\lambda)^{3}\right] & \text { if } 0 \leqslant r<R-\lambda, \\ \frac{\rho\left(R^{2}-r^{2}\right)}{6 \varepsilon}+\frac{\rho}{3 \varepsilon}(R-\lambda)^{3}\left(\frac{1}{R}-\frac{1}{r}\right)+\frac{\rho}{3 \varepsilon_{0} R}\left[R^{3}-(R-\lambda)^{3}\right] & \text { if } R-\lambda \leqslant r \leqslant R,\end{cases}
$$




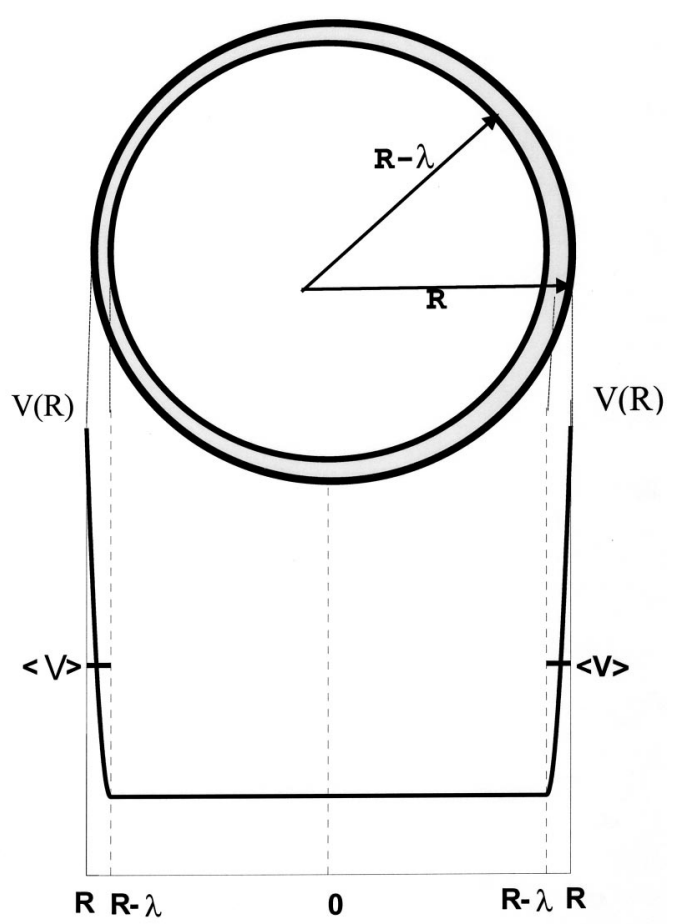

FIG. 1. Profile of the electric potential inside a grain as a result of the density of charge.

where $\varepsilon_{0}$ is the dielectric constant of vacuum. Notice that the electric potential is a constant along the interval $0 \leqslant r<R-\lambda$. From now on, we will denote to this constant value as $V_{\text {int }}$. Making use of Eq. (4), two useful results can be obtained. First, the electric potential at the interface $V(R)$ is straightforwardly calculated by substitution of $r=R$ in Eq. (4). That will give us the following expression:

$$
V(R)=\frac{\rho R^{2}}{3 \varepsilon_{0}}\left[1-\left(1-\frac{\lambda}{R}\right)^{3}\right]
$$

In the limiting case $\lambda \ll R$, a Taylor expansion of Eq. (5) up to the first order in $\lambda / R$ will yield

$$
V(R) \cong \frac{\rho R \lambda}{\varepsilon_{0}} \quad \text { at } r=R \text {; for } \lambda \ll R .
$$

Second, the mean value of the electric potential $(\langle V\rangle)$ in the interval $R-\lambda \leqslant r \leqslant R$ is usually defined as

$$
\langle V\rangle=\frac{1}{\lambda} \int_{R-\lambda}^{R} V(r) d r .
$$

The mean value can be calculated from Eq. (4); and after the Taylor expansion, retaining the lowest order in $\lambda / R$, the following results:

$$
\langle V\rangle=V_{\text {int }}-\frac{1}{6} \frac{\rho R^{2}}{\varepsilon}\left(\frac{\lambda}{R}\right)^{2} .
$$

The dependence of $V(r)$ with the distance to the grain center is plotted in Fig. 1, as well as the values of $V(R)$ and $\langle V\rangle$. Equations (6) and (8) will be repeatedly used along this model.

\section{Constitutive equations for creep}

Consider the application of a uniaxial mechanical stress: the only nonzero stress component of the stress tensor is $\tau_{33}=\sigma$. In the framework of the diffusional creep models, deformation takes place as a consequence of the existence of mass fluxes induced by the gradient of the chemical potential around the grain. As a final result, the mass fluxes generated in the steady state are responsible for the constant strain rate measured during plastic deformation at a constant stress.

If a local electric field is present close to the grain boundaries, and the charge carriers responsible for diffusion are depleted, a significant creep resistance would be expected. In the case of YTZP, the charge carrier controlling diffusion, and plastic deformation, is $\mathrm{Zr}^{4+}$ (see Refs. 4 and 17-19). These cations tend to locate in the regions where the electric potential reaches a minimum; i.e., around the grain center. Consequently, the grain-boundary concentration of $\mathrm{Zr}^{4+}$ becomes smaller. The diffusion coefficient of these cations from the center to the grain boundaries should decrease due to the presence of the local electric field next to the grain boundaries. We shall refer to the magnitudes linked to this cation with the subscript " $D$ " from now on.

In the case of local electric potential resulting from segregation, local concentration can be related to the local electric potential. In effect, according to thermodynamics, in the limit of dilute solid solutions, the chemical potential of one component has the following dependence on its concentration and electric potential: ${ }^{20}$

$$
\mu_{D}(c, T, \phi)=\mu_{D}^{0}+k T \ln \left(\frac{c_{D}(r)}{c_{D}(0)}\right)+z_{D} e\left[V(r)-V_{\text {int }}\right]
$$

where $z_{D}$ is the valence of the component, $e$ is the electron charge, and $\mu_{D}^{0}$ is the chemical potential at the center of the grain [where no segregation exists, and the concentration of the component is $\left.c_{D}(0)\right]$. As denoted previously, $V_{\text {int }}$ is the electric potential in the region where there is no segregation. When equilibrium inside the grains is reached, $\mu_{D}=\mu_{D}^{0}$ and hence

$$
c_{D}(r)=c_{D}(0) \exp \left(\frac{-z_{D} e\left[V(r)-V_{\text {int }}\right]}{k T}\right) .
$$

Assume that Eq. (10) is valid during deformation even though the plastic deformation is not a thermodynamical state of equilibrium. This assumption is made in all diffusional creep models.

We are going to proceed according to the standard procedure developed in the Nabarro-Herring $(\mathrm{NH})$ model and the extension carried out for segregation in a monodimensional system. ${ }^{3,21}$ The generalization for our three-dimensional system is described in the Appendix. According to the $\mathrm{NH}$ model, the creep equation can be written as

$$
\dot{\varepsilon}=\frac{16}{3} \frac{\sigma \Omega}{k T d^{2}} D_{\mathrm{eff}} \alpha,
$$


where $D_{\text {eff }}$ is the effective diffusion coefficient of the diffusing species taking into account all the possible diffusion paths, $d=2 R$ is usually called as the "grain size," and $\alpha$ is given by

$$
\alpha=\frac{R^{2} / 2}{\int_{0}^{R} \frac{c_{D}(0)}{c_{D}(r)} r d r} .
$$

Equations (11) and (12) are the key equations to critically analyze the physical implications of segregation. The factor labeled as " $\alpha$ " plays a major role in determining the behavior of the system. In effect, if we consider a system in which no segregation exists; i.e., $c(r)=c_{0}$; then no depleted thin layer is found in the grains, $\alpha=1$, and (11) takes the form

$$
\dot{\varepsilon} \cong \frac{16}{3} \frac{\sigma \Omega}{k T d^{2}} D_{\text {eff }}
$$

which is nothing but the well-known result obtained by Nabarro and Herring in their classical work; (Notice that the proportionality constant depends on the geometry of grains. For other grain shapes, values as high as 40 are reported.)

A significant change is obtained if the carrier concentration controlling both diffusion and so plasticity is depleted. If this is the case, $c_{D}(r)$ is significantly reduced in a thin layer close to the grain boundary, and $\alpha<1$, in consequence, the strain rate would be much smaller, i.e.; the creep resistance is improved.

In accordance with Eq. (10), the parameter $\alpha$ [Eq. (12)] can be evaluated by means of the equation

$$
\begin{aligned}
\alpha & =\frac{R^{2} / 2}{\int_{0}^{R} r \exp \left(\frac{z_{D} e\left[V(r)-V_{\text {int }}\right]}{k T}\right) d r} \\
& \cong \frac{R^{2} / 2}{\frac{1}{2}(R-\lambda)^{2}+\int_{R-\lambda}^{R} r \exp \left(\frac{z_{D} e\langle V\rangle-z_{D} e V_{\text {int }}}{k T}\right) d r} .
\end{aligned}
$$

In this equation, it is assumed that $\lambda \ll R$ so that the electric potential can be approximated by its mean value $\langle V\rangle$ in the region $R-\lambda<r<R$.

After some algebra, it is possible to write the following expression for $\alpha$ :

$$
\begin{aligned}
\alpha & \cong \frac{1}{1+4 \frac{\lambda}{d}\left[\exp \left(\frac{z_{D} e\langle V\rangle-z_{D} e V_{\text {int }}}{k T}\right)-1\right]} \\
& =\frac{1}{1+4 \frac{\lambda}{d}\left[\exp \left(\frac{-z_{D} e \rho \lambda^{2}}{6 \varepsilon k T}\right)-1\right]} .
\end{aligned}
$$

Finally, making use of Eq. (6), it is possible to eliminate the charge density in Eq. (15) and the substitution of this last equation for $\alpha$ into Eq. (11) allows us to conclude that the constitutive equation for creep is

$$
\dot{\varepsilon}=\frac{16}{3} \frac{\sigma \Omega}{k T d^{2}} \frac{D_{\text {eff }}}{1+4 \frac{\lambda}{d}\left[\exp \left(\frac{-z_{D} e V(R)}{3 \varepsilon_{r} k T} \frac{\lambda}{d}\right)-1\right]},
$$

where $\varepsilon_{r}=\varepsilon / \varepsilon_{0}$ is the relative dielectric constant of the material under study.

\section{Generalization for the case of a grain-boundary sliding controlling mechanism accommodated by diffusion}

In the preceeding section, the diffusion creep model has been used for the sake of simplicity as a starting point for the development of our model. However, the same reasoning can be repeated if a model in which grain-boundary sliding (GBS) is the deformation-controlling mechanism. In effect, diffusion is the rate-controlling process ${ }^{22}$ even in the GBS's in order to maintain the contact between grains while deformation takes place. In the GBS model, deformation is determined by the relative displacement between grains. The stress is relieved, and the subsequent condition of contact between grains is obtained by the flux of matter. This transport of matter is driven by the gradient of chemical potential. Once the chemical potential is calculated, the same reasoning applied in the previous sections can be repeated yielding an effective diffusion coefficient. In the case of GBS in metals, Sherby and Wadsworth ${ }^{23}$ found that the constitutive creep equation is given by

$$
\dot{\varepsilon}=A \frac{G b}{k T}\left(\frac{\sigma}{G}\right)^{2}\left(\frac{b}{d}\right)^{2} D_{\text {eff }},
$$

where $A$ is an empirical constant, $G$ is the shear modulus, and $D_{\text {eff }}$ is the effective diffusion coefficient. Equation (17) is extended for the case of YTZP according to the following expression given below:

$$
\dot{\varepsilon}=2 \times 10^{7} \frac{G b}{k T}\left(\frac{\sigma-\sigma_{\mathrm{TS}}}{G}\right)^{2}\left(\frac{b}{d}\right)^{2} D_{\text {latt }}^{\mathrm{Zr}},
$$

where $b$ is the Burgers vectors of dislocations in zirconia, $\sigma_{\mathrm{TS}}$ is a threshold stress for plasticity, and $D_{\text {latt }}^{\mathrm{Zr}}$ is the lattice diffusion coefficient for zirconium. Equation (18) has been confirmed experimentally and analyzed thoroughly by Jiménez-Melendo et $a .^{4}{ }^{4}$ for YTZP.

The extension for nanoceramics can be made with the following statements: First, the threshold stress is negligible in the case of YTZP nanoceramics. ${ }^{24}$ Second, the effect of segregation has been proved to be a modification of the effective diffusion coefficient controlling GBS accommodation. This effect is quantitatively described by the factor $\alpha$ [see Eq. (15)].

Consequently, it is reasonable to accept the following expression for the constitutive creep equation of TZP nanoceramics:

$$
\dot{\varepsilon}=\frac{2 \times 10^{7}}{1+4 \frac{\lambda}{d}\left[\exp \left(\frac{-z_{D} e V(R)}{3 \varepsilon_{r} k T} \frac{\lambda}{d}\right)-1\right]} \frac{G b}{k T}\left(\frac{\sigma}{G}\right)^{2}\left(\frac{b}{d}\right)^{2} D_{\text {latt }}^{\mathrm{Zr}} \text {. }
$$




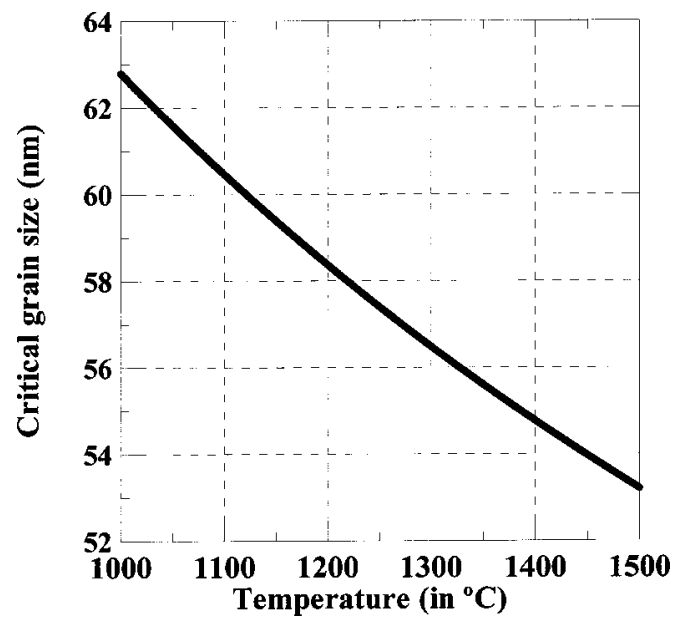

FIG. 2. The critical size, or maximum value of the grain size at which the segregation effects have still some influence on mechanical behavior, as a function of temperature.

Notice that Eq. (19) converges to Eq. (18) when $d \gg \lambda$ and $\sigma \gg \sigma_{\mathrm{TS}}$. In order to compare the mechanical behavior of YTZP nanoceramics (in which $\sigma_{\mathrm{TS}} \cong 0$ ) and conventional submicrometric YTZP polycrystals (in which $\sigma_{\mathrm{TS}}$ does not vanish at all), all reported data will correspond to the highstress limit, $\sigma \gg \sigma_{\mathrm{TS}}$.

\section{RESULTS AND DISCUSSION}

Equation (19) is the constitutive equation for creep in materials where a local electric potential due to segregation of cations with an effective ionic charge at the grain boundaries plays a significant role in high-temperature diffusion. A rigourous analysis of its range of validity, as well as the implications on the plastic deformation parameters can be performed. First of all, it is essential to notice that no adjustable parameters are required to make quantitative estimations. Only the potential $V(R)$ and the thickness of the depletion layer $\lambda$ are required. Both parameters can be experimentally determined. Experimental inputs for YTZP (Refs. 25-27) are summarized in Table I.

\section{A. Range of validity of this model}

In general, the effect of the segregation layer is enhanced if either temperature or the average grain size is decreased. Assuming that a quantitative estimation of the range of temperatures and grain sizes at which a significant deviation from the $\lambda / d \rightarrow 0$ limit takes place is

$$
\frac{\Delta \dot{\varepsilon}}{\dot{\varepsilon}} \leqslant 0.10
$$

This arbitrary assumption restricts the relative deviation from the $\lambda / d \rightarrow 0$ limit up to $10 \%$. Using Eq. (19) gives rise to

$$
4 \frac{\lambda}{d}\left[\exp \left(\frac{-z_{D} e V(R)}{3 \varepsilon_{r} k T} \frac{\lambda}{d}\right)-1\right] \leqslant 0.10 .
$$

Application of this assumption in YTZP is shown in Fig. 2.
TABLE I. Experimental inputs needed for determination of the theoretical results in the case of YTZ.

\begin{tabular}{lcc}
\hline \hline Quantity & Experimental values & $\begin{array}{c}\text { Value adopted } \\
\text { in this work }\end{array}$ \\
\hline $\begin{array}{l}\text { Relative dielectric } \\
\text { constant }(\varepsilon r)\end{array}$ & $\begin{array}{c}4.58-4.71 \\
\text { (see Refs. 25 and 26) }\end{array}$ & 4.65 \\
$\begin{array}{l}\text { Thickness of the } \\
\text { deplectionlayer } \\
(\lambda, \text { in nm) }\end{array}$ & $2-10$ (see Ref. 11) & 5 \\
$\begin{array}{l}\text { Electric potential at } \\
\text { the grain boundaries } \\
{[e \phi(R) \text { in eV] }}\end{array}$ & $\begin{array}{c}\text { Between }-1 \text { and }-2 \\
\text { (see Ref. 27) }\end{array}$ \\
\hline \hline
\end{tabular}

The critical grain size (i.e., the minimum value of the grain size at which the micron-sized behavior is still measured) versus the temperature is shown in that figure. It is clear that this critical size is around $60 \mathrm{~nm}$ and is not very sensitive to temperature. The dependence with temperature is consistent with the fact that segregation effects tend to vanish when temperature rises. Notice that, for values of the grain size smaller than the critical value at a fixed temperature, the deviation is much higher than $10 \%$. The critical value given here defines the upper limit for observation of segregation effects at the current temperature. In effect, for $1200^{\circ} \mathrm{C}$ and an average grain size equal to $50 \mathrm{~nm}$, the relative deviation (20) is higher than $55 \%$. This means that a remarkable hardening effect should be expected for such conditions. This fact is in agreement with the data reported by Gutiérrez-Mora, Jiménez-Melendo, Domínguez-Rodríguez, and Chaim, ${ }^{7}$ in which the predicted hardening effect has been experimentally observed.

\section{B. Influence on mechanical parameters}

\section{Grain-size exponent}

The grain-size exponent is defined as

$$
p=-\left(\frac{\partial \ln \dot{\varepsilon}}{\partial \ln d}\right)_{T, \sigma} .
$$

For diffusional creep models $p=2$ (or 3 if diffusion along grain boundaries becomes important). This is usually observed in YTZP superplastic ceramics in the submicron range. In order to explore the predictions of our model, the derivative given in Eq. (22) was performed for the case of our constitutive equation, yielding the following result:

$$
p=2-\frac{4 \frac{\lambda}{d}\left[1-\frac{\lambda}{d} \frac{z_{D} e V(R)}{3 \varepsilon_{r} k T}\right] \exp \left(\frac{-1}{3 \varepsilon_{r}} \frac{\lambda}{d} \frac{z_{D} e V(R)}{k T}\right)-4 \frac{\lambda}{d}}{1+4 \frac{\lambda}{d}\left[\exp \left(\frac{-1}{3 \varepsilon_{r}} \frac{z_{D} e V(R)}{k T} \frac{\lambda}{d}\right)-1\right]} .
$$

The exponent can be calculated using values from Table I. The results are shown in Fig. 3. The $p$ exponent decreases with decreasing grain size: from the expected value of 2 


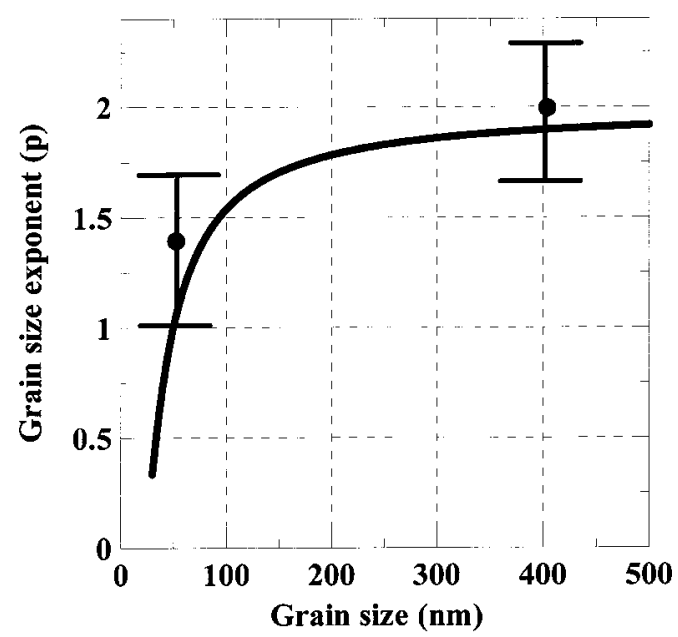

FIG. 3. Predicted grain-size exponent versus the grain size at $1200{ }^{\circ} \mathrm{C}$ for YTZP. Experimental values (Refs. 4 and 6) are also plotted in the figure.

usually measured for submicron YTZP ceramics, to values close to 1 for grain sizes lower than $50 \mathrm{~nm}$. This prediction is in excellent agreement with the experimental data measured by Jiménez-Melendo, Domínguez-Rodríguez, and Bravo-León ${ }^{4}$ (for submicron YTZP) and DomínguezRodríguez et al. ${ }^{6}$ in which a $p$ value of 1.4 was found for $d$ around $50 \mathrm{~nm}$ at $1200^{\circ} \mathrm{C}$ (Fig. 3).

\section{Stress exponents $(n)$ and activation energies $(Q)$}

These magnitudes are defined as follows:

$$
\begin{gathered}
n=\left(\frac{\partial \ln \dot{\varepsilon}}{\partial \ln \sigma}\right)_{T, d}, \\
Q=-\left(\frac{\partial \ln \dot{\varepsilon}}{\partial \beta}\right)_{\sigma, d},
\end{gathered}
$$

where $\beta=1 / k T$. According to our model, $n$ is not a function of the grain size " $d$." This means that $n$ should be 2 for superplastic behavior (or 1 if the $\mathrm{NH}$ model is operating at very high temperatures) independent of grain size. This seems to be the case in YTZP.

The activation energy does, however, depend on grain size. After algebraic operation, it can be shown that

$$
Q=Q_{D}-\frac{\frac{4}{3 \varepsilon_{r}}\left(\frac{\lambda}{d}\right)^{2} z_{D} e V(R) \exp \left(\frac{-1}{3 \varepsilon_{r}} \frac{\lambda}{d} \frac{z_{D} e V(R)}{k T}\right)}{1+4 \frac{\lambda}{d}\left[\exp \left(\frac{-1}{3 \varepsilon_{r}} \frac{\lambda}{d} \frac{z_{D} e V(R)}{k T}\right)-1\right]},
$$

where $Q_{D}$ is the activation energy of the Arrhenius dependence of the cationic diffusion coefficient. DomínguezRodríguez et al. ${ }^{6}$ have reported a dependence of $Q$ with the grain size. In their results, $Q \cong 7.0 \pm 0.2 \mathrm{eV} /$ atom for $d$ $\cong 50 \mathrm{~nm}$; and it decreases to $Q \sim 5.2 \pm 0.2 \mathrm{eV} /$ atom for submicron YTZP ceramics. This last value has been also reported by Jiménez-Melendo, Domínguez-Rodríguez, and

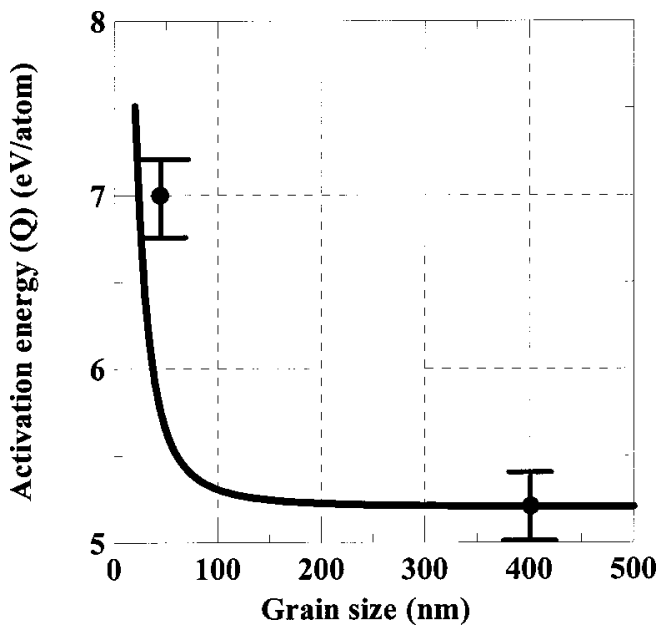

FIG. 4. Predicted activation energy versus the grain size at $1200{ }^{\circ} \mathrm{C}$ for YTZP. Experimental results (Refs. 4 and 6) are plotted also.

Bravo-León ${ }^{4}$ in submicron YTZP specimens in the limit $\sigma$ $\gg \sigma_{\mathrm{TS}}$. These data are in reasonable agreement with the predictions of the model (Fig. 4). Very recently, Lorenzo-Martín et $a l^{28}$ have reported the same tendency in YTP nanocrystals deformed at $1150{ }^{\circ} \mathrm{C}$ with grain size around $50 \mathrm{~nm}$, even when a certain amount of a glassy phase is present.

\section{Strain rate dependence with the grain size}

As cited previously, a significant improvement in creep resistance has been reported. ${ }^{7,29}$ Experimentally it has been observed that the strain rate normalized by the grain size $\left(\dot{\varepsilon} d^{2}\right)$ decreases one order of magnitude when the grain size is approximately $40 \mathrm{~nm}$ (see Ref. 7). This can be qualitatively explained by means of the model: the value of $\alpha$ in the creep equation [Eq. (16)] gives rise to a significant hardening (the normalized strain rate is one order of magnitude lower than expected) for grain sizes lower than $40 \mathrm{~nm}$ (horizontal line in Fig. 5). However, the numerical agreement is not

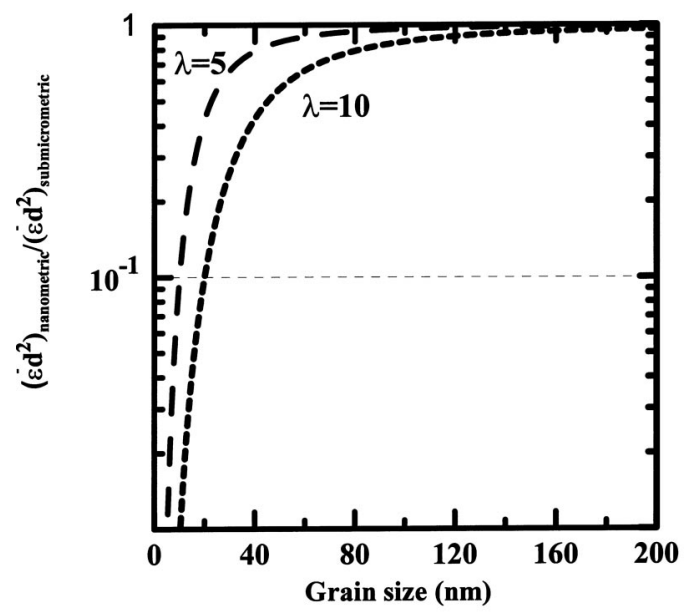

FIG. 5. Strain rate versus the grain size predicted by this model for two different values of $\lambda$. The horizontal line corresponds to the hardening found experimentally for nano-YTZP $(d=50 \mathrm{~nm})$. 
completely satisfactory. This disagreement can be due to two facts: (i) The hardening is quite sensitive to the value of the screening length $\lambda$. This is emphasized in Fig. 5 in a second plot for the hardening expected for $\lambda=10 \mathrm{~nm}$. In this second case, the theoretical predictions are closer to the experimental results. Moreover, the Debye screening length is a function of temperature (with a $T^{1 / 2}$ dependence). ${ }^{3}$ As the experimental values of $\lambda$ are determined at room temperature, a more accurate comparison should be made if the temperature correction is introduced into the equations. When this is made, the agreement with the experimental results is much better for the hardening ratio, as well as both the $p$ and $Q$ experiments. (ii) It is important to note that the model predicts a very pronounced dependence of the hardening ratio versus the grain size that implies that an accurate comparison can be made only if a wide range of mean grain sizes are measured. This should be a future endeavor.

It is essential to realize that the origin of this hardening effect is not due to the change of the grain-size exponent. Besides, if the origin of this effect were the change in the value of $p$ from 2 to 1.4 as reported (see Ref. 7), the expected tendency would be an increase of the strain rate, in contradiction with the existence of the hardening effect. This fact shows clearly that the origin of the hardening is only understood by the change in the effective diffusion coefficient as a result of segregation. Mathematically, it is the term $\alpha$ that is responsible for this change in the diffusion coefficient that accounts for the hardening.

\section{CONCLUSIONS}

A model for high-temperature plastic deformation of polycrystals has been proposed. This model introduces the effect of the existence of a charged layer of segregated dopants at the grain boundaries on diffusion, and in consequence, on high-temperature plasticity. The model predicts a grain-size exponent (for $d \leqslant 50 \mathrm{~nm}$ ) $p<2$. The model has been compared to experimental results for YTZP nanocrystals. It agrees quantitatively with most of the hightemperature plasticity features. Future work should be conducted in other ceramics to validate experimentally the model in more cases.

\section{ACKNOWLEDGMENTS}

The authors would like to acknowledge financial support by the Spanish Ministerio de Ciencia y Tecnología through Project No. MAT2000-0622. We also acknowledge the kind help of Professor Jules Routbort (Argonne National Laboratory, Illinois) during the writing of the manuscript. His corrections of the English style and his suggestions on some of the physical assumptions made in the model have certainly improved the quality of our work.

\section{APPENDIX}

In the framework of the $\mathrm{NH}$ model, deformation takes place as a consequence of the existence of mass fluxes induced by the gradient in the chemical potential all around the grain. For the case of a perfectly spherical grain, the mass flux along the compression axis $\left[J_{\mathrm{II}}(r, \theta=0)\right]$ is given by

$$
\int_{0}^{R} J_{\mathrm{II}}(r, \theta=0) \frac{k T X_{D}}{c_{D}(r) D_{\mathrm{eff}}} d r=\mu(0, \theta=0)-\mu(R, \theta=0),
$$

where $c_{D}$ is the number of lattice defects responsible for deformation per unit volume, $X_{D}$ is the molar fraction of the controlling species, $J_{\mathrm{II}}(r, \theta=0)$ is the mass flux along the loading axis, $D_{\text {eff }}$ is the effective diffusion coefficient (in which all the different diffusion paths are considered), ${ }^{30}$ and $\mu$ is the chemical potential of the whole system. [Notice that the mass flux and the chemical potential should be a function of $r$ and $\theta$. The loading axis is a rotation symmetry axis. In consequence, the mass flux is not spherically symmetric, as expected, because the existence of a uniaxial loading axis breaks the spherical symmetry of the system. The strain rate is controlled by the deformation along the loading axis. This fact justifies the use of the $J_{\mathrm{II}}(r, \theta=0)$ in Eq. (A1).] Notice that the integral in Eq. (A1) is extended from the center to the surface along the diameter parallel to the loading axis. Equation (1) is an integral version of the general Fick diffusion law. ${ }^{31}$

If the chemical potential is known inside the grain as a function of the position, the mass flux can be calculated rigorously, and this one can be related to the strain rate of the system. The chemical potential must satisfy the Laplace equation, with the boundary condition given below: ${ }^{21}$

$$
\mu(R, \theta)=\mu_{0}-\sigma_{n} \Omega_{D}=\mu_{0}-\sigma \Omega_{D} \cos ^{2} \theta,
$$

where $\Omega_{D}$ is the atomic volume of the lattice defect involved in the diffusion process, and $\sigma_{n}$ is the normal component of the stress on the surface grain.

It is easy to verify that the solution for the chemical potential in agreement with the boundary conditions imposed in Eq. (A2) is

$$
\mu=\mu_{0}+\frac{1}{3} \frac{\sigma \Omega_{D}}{R^{2}}\left(r^{2}-R^{2}\right)-\frac{\sigma \Omega_{D}}{R^{2}} r^{2} \cos ^{2} \theta .
$$

There is a relationship between the mass flux on the surface (at $r=R$ ) and the strain rate: ${ }^{20}$

$$
X_{D} J_{\mathrm{II}}(R, \theta=0)=R c_{D}(0) \dot{\varepsilon} .
$$

Equation (A1) can be rewritten as function of the mass flux at the boundary of the grain if the following condition is taken into account:

$$
J_{\mathrm{II}}(r, \theta=0)=\frac{J_{\mathrm{II}}(R, \theta=0)}{R} r .
$$

This condition is obtained as a consequence of the fact that $\nabla \cdot \mathbf{j}=0$ (see Ref. 30) resulting in

$$
\begin{aligned}
\int_{0}^{R} \frac{J_{\mathrm{II}}(R)}{R} r \frac{k T X_{D}}{c_{D}(r) D_{\mathrm{eff}}} d r & =\frac{J_{\mathrm{II}}(R)}{R} \frac{k T X_{D}}{c_{D}(0) D_{\mathrm{eff}}} \int_{0}^{R} r \frac{c_{D}(0)}{c_{D}(r)} d r \\
& =\mu(0,0)-\mu(R, 0) .
\end{aligned}
$$


A direct use of Eq. (A6) with Eqs. (A5) and (A1) gives rise to the following result:

$$
\dot{\varepsilon}=\frac{16}{3} \frac{\sigma \Omega_{D}}{k T d^{2}} D_{\mathrm{eff}} \alpha
$$

where $d=2 R$ and $\alpha$ is given by

*Corresponding author. Email address: adorod@us.es

${ }^{1}$ H. Gleiter, J. Weissmüller, O. Wollersheim, and R. Würschum, Acta Mater. 49, 737 (2001).

${ }^{2}$ I. Chen and L. An Xue, J. Am. Ceram. Soc. 73, 2585 (1990).

${ }^{3}$ J. Jamnick and R. Raj, J. Am. Ceram. Soc. 79, 196 (1996).

${ }^{4}$ M. Jiménez-Melendo, A. Domínguez-Rodríguez, and A. BravoLeón, J. Am. Ceram. Soc. 81, 2761 (1998).

${ }^{5}$ M. Jiménez-Melendo and A. Domínguez-Rodríguez, Acta Mater. 79, 1591 (2000).

${ }^{6}$ A. Domínguez-Rodríguez, F. Gutiérrez-Mora, M. JiménezMelendo, J. L. Routbort, and R. Chaim, Mater. Sci. Eng., A 302, 154 (2001).

${ }^{7}$ F. Gutiérrez-Mora, M. Jiménez-Melendo, A. DomínguezRodríguez, and R. Chaim, Key Eng. Mater. 171-74, 787 (2000).

${ }^{8}$ G. Theunissen, A. Winnubst, and A. Burggraaf, J. Mater. Sci. 27, 5057 (1992).

${ }^{9}$ J. Hines, Y. Ikuhara, A. Chokshi, and T. Sakuma, Acta Mater. 46, 5557 (1998).

${ }^{10}$ S. Stemmer, J. Vleugels, and O. Van der Biest, J. Eur. Ceram. Soc. 18, 1565 (1998).

${ }^{11}$ P. Flewit and R. Wild, Grain Boundaries: Their Microstructure and Chemistry (John Wiley \& Sons Ltd., Chichester, U.K., 2001), p. 137.

${ }^{12}$ H. Conrad and D. Yang, Acta Mater. 48, 4045 (2000).

${ }^{13}$ H. Conrad, Scr. Mater. 44, 311 (2001).

${ }^{14}$ L. Pauling, The Nature of the Chemical Bond, 3rd ed. (Cornell University Press, Ithaca, NY, 1960), p. 514.

${ }^{15}$ F. Wakai, T. Nagano, and T. Iga, J. Am. Ceram. Soc. 80, 2361 (1997)

${ }^{16}$ J. D. Jackson, Classical Electrodynamics, 3rd Ed. (John Wiley \&
Sons, Inc., New York, 1998), p. 167.

${ }^{17}$ A. Domínguez-Rodríguez, A. H. Heuer, and J. Castaing, Radiat. Eff. Defects Solids 119-121, 759 (1991).

${ }^{18}$ J. Martínez-Fernández, M. Jiménez-Melendo, A. DomínguezRodríguez, and A. H. Heuer, J. Am. Ceram. Soc. 73, 2452 (1990).

${ }^{19}$ D. Gómez-García, J. Martínez-Fernández, A. DomínguezRodríguez, P. Eveno, and J. Castaing, Acta Metall. Mater. 44, 991 (1996).

${ }^{20}$ R. T. DeHoff, Thermodynamics in Materials Science (McGrawHill International, Singapore, 1993), p. 187.

${ }^{21}$ C. Herring, J. Appl. Phys. 21, 437 (1950).

${ }^{22}$ I. M. Lifshitz, Sov. Phys. JETP 17, 909 (1963).

${ }^{23}$ O. D. Sherby and J. Wadsworth, Prog. Mater. Sci. 33, 169 (1989).

${ }^{24}$ D. Gómez-García, C. Lorenzo-Martín, A. Muñoz-Bernabé, and A. Domínguez-Rodríguez, Philos. Mag. B 83, 93 (2003).

${ }^{25}$ D. J. Green, R. H. Hannick, and M. V. Swain, Transformation Toughening in Zirconia (CRC, Boca Raton, FL, 1989), p. 221.

${ }^{26}$ R. Stevens, Zirconia and Zirconia Ceramics (Magnesium EleKtron Ltd., Litho, Twickenham, U.K., 2000), p. 2.

${ }^{27}$ S. Hwang and I. Chen, J. Am. Ceram. Soc. 73, 3269 (1990).

${ }^{28}$ C. Lorenzo-Martín, D. Gómez-García, A. Muñoz-Bernabé, A. Domínguez-Rodríguez, D. XueMing, and J. Gómez-Herrero, J. Eur. Ceram. Soc. 22, 2603 (2002).

${ }^{29}$ F. Gutiérrez-Mora, A. Domínguez-Rodríguez, and M. JiménezMelendo, J. Eur. Ceram. Soc. 22, 2615 (2002).

${ }^{30}$ J. Philibert, Solid State Ionics 12, 321 (1984).

${ }^{31}$ J. Philibert, Diffusion et Transport de Matière dans les Solides (Les Éditions de Physique, Les Ulis Cedex, France, 1990), p. 450 . 